\title{
Endovascular Management of an Acquired Subclavian Arteriovenous Fistula Secondary to Chronic Venous Occlusion after Pacemaker Insertion
}

1) Department of Diagnostic and Interventional Radiology, Osaka University Graduate School of Medicine, Osaka, Japan

\author{
Matthew W. Lukies ${ }^{1)}$, Keigo Osuga ${ }^{1)}$, Tetsuro Nakazawa ${ }^{1)}$, Yusuke Ono ${ }^{1)}$, Hiroki Higashihara ${ }^{1)}$, \\ Noriyuki Tomiyama ${ }^{1)}$
}

\begin{abstract}
Subclavian arteriovenous (AV) fistula formation is a rare complication of chronic deep vein thrombosis after pacemaker insertion. Here we present the case of an 80-year-old woman who developed a symptomatic arteriovenous fistula more than two years after cardiac pacemaker insertion, as a result of chronic thrombotic stenosis of the left subclavian vein. The defect was managed endovascularly by embolization, balloon angioplasty, and stent graft insertion, in order to both close the fistula and recanalize the subclavian vein to prevent recurrence. In this case report we present the endovascular procedure undertaken, followed by a discussion of the relevant pathophysiology, treatment options, and approach considerations.
\end{abstract}

Key words: Cardiac pacemaker, AV fistula, endovascular intervention, stent graft

(Interventional Radiology 2017; 2: 33-37)

\section{Introduction}

Iatrogenic arteriovenous fistula (AVF) is estimated to occur at a rate of $0.58 \%$ as a complication of venous catheterization [1]. The fistula is commonly formed at the time of the procedure as a consequence of direct vessel trauma. AVF formation as a long-term complication of venous thrombosis is rare, but has been reported in the lower limbs [2, 3]. Although venous thrombosis is a relatively common complication after cardiac device insertion, it rarely causes severe symptoms or further complications because of the establishment of collateral flow [4].

Treatment options for acquired AVFs include surgical ligation, embolization, sclerotherapy, and stent graft insertion $[5,6]$. The treatment of traumatic AVFs with stent grafts is well established [7], but few reports have covered the treatment of arteriovenous fistulas caused by chronic venous occlusion. The treatment approach must consider both closure of the fistula and recanalization of the chronic ve- nous occlusion to prevent further angiogenesis and AVF formation.

Here we present a case of pacemaker-induced iatrogenic chronic subclavian venous obstruction causing acquired AVF, which was treated endovascularly with embolization, recanalization, and stent graft insertion.

\section{Case Report}

\section{Patient}

An 80-year-old woman presented with a history of progressive left upper limb swelling and pain for 1-2 years. Past medical history included sick sinus syndrome with pacemaker implantation four years previously, type two diabetes mellitus, and laparoscopic uterine myomectomy. The patient stated that the cardiac pacemaker insertion procedure was uncomplicated, although actual medical records from the procedure were unavailable. An intravenous contrastenhanced CT scan (Figure 1) identified left subclavian vein 


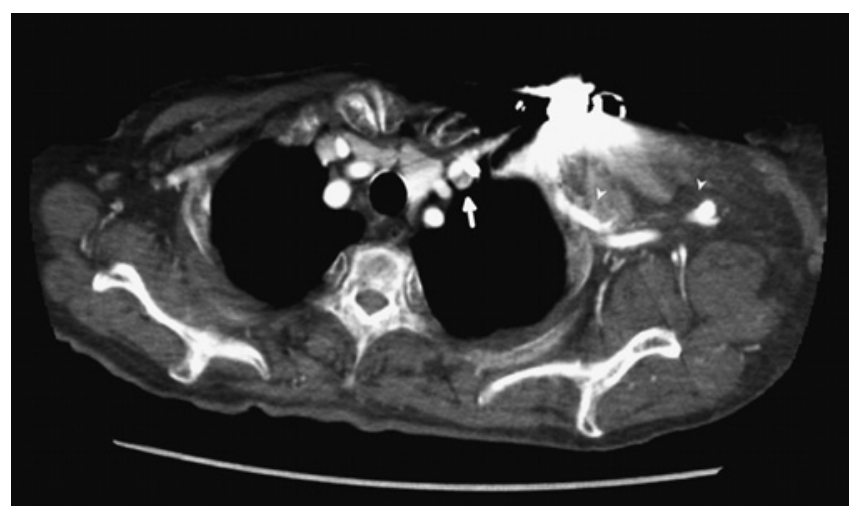

Figure 1. Intravenous contrast-enhanced CT. Thrombus observed in the left subclavian vein (arrow), raising suspicion of occlusion; the distal subclavian and axillary veins (arrowheads) are enhanced because of backflow of venous blood from a possible arteriovenous fistula.

thrombosis and possible occlusion, but the symptoms were mild and the patient was managed conservatively with continuation of warfarin therapy and follow up.

Over subsequent months the swelling and pain worsened, and ultrasound revealed a complex subclavian AVF involving small vessels from the left distal subclavian artery to the left distal subclavian vein, with retrograde flow through to the axillary vein. The patient was then referred to Interventional Radiology for endovascular management.

\section{Procedure}

Arterial digital subtraction angiography (DSA) was performed via a left femoral approach (INR 1.67 at time of procedure) and demonstrated multiple small fistulas from the subclavian artery directly connecting to the main trunk of the subclavian vein (Figure 2. A). Occlusion of the subclavian vein was noted, and there was retrograde flow through the vein distally to the axillary vein. Five of the feeder branches were embolized with a total of $1.4 \mathrm{~mL}$ of $50 \% \mathrm{~N}$ butyl cyanoacrylate (NBCA) mixed with iodized oil (Figure 2. B, C). This led to a reduction in the blood flow through the AVFs, but multiple small shunts remained (Figure 2. D).

Venous angiography was then performed via a right femoral approach and demonstrated complete occlusion of the left subclavian vein (Figure 2. E). However, it was difficult to pass through the occlusion using a 0.035 -inch hydrophilic guidewire, so alternative access was obtained via the left cephalic vein and injection of contrast revealed a short segment of occlusion. Venous angioplasty was performed using non-compliant balloons (Mustang $4 \times 20 \mathrm{~mm}$ at 10-22 atm, and $6 \times 20 \mathrm{~mm}$ at 12-22 atm, Boston Scientific Japan, Tokyo, Japan) via a through-through method from the femoral vein, given the bleeding risk associated with using a larger size sheath in the cephalic vein receiving arterial pressure from the AVF located deeply in the markedly swollen arm (Figure 2. F). The occlusion in the subclavian vein was then recanalized, but numerous small shunts and retrograde flow to the axillary vein still remained on arterial DSA.
Given the possibility of interference with the cardiac pacemaker leads, we were reluctant to place a stent graft in the occluded vein. Instead, we decided to place a stent graft via a left femoral approach into the left subclavian artery. Because of the short length of the available delivery catheter, more proximal left femoral artery access was obtained by surgical cutdown, and an $8 \times 60 \mathrm{~mm}$ stent graft (Fluency, Medicon Inc., Osaka, Japan) was inserted over the site of the AVFs in the left subclavian artery to minimize the inflow of the remaining small shunts (Figure 2. G). The number and size of the shunts decreased and retrograde venous flow was corrected (Figure 2. H). Venous DSA from a cephalic approach demonstrated restoration of antegrade venous flow (Figure 2. I).

\section{Outcome and follow up}

At the time of the procedure, the patient had significant venous backflow and pooling in her left arm, causing progressive swelling and pain (Figure 3. A). Two weeks after the procedure the discoloration and symptoms had significantly improved (Figure 3. B). Around nine months later, the swelling and discoloration had partially recurred (Figure 3. C) and the patient underwent a second venoplasty procedure to recanalize the subclavian vein. A total of three venoplasty procedures have been performed over the two and half years since the first DSA to maintain patency of the subclavian vein and it is likely that further procedures will be required (Figure 3. D).

\section{Discussion}

While trauma, either accidental or procedure-related, is the main cause of acquired AVF, other causes have been recognized, such as venous occlusion and angiogenesis. The estimated incidence of iatrogenic venous catheter-induced AVF is $0.58 \%$ [1], based on a review of 470 subclavian and 49 internal jugular vein catheter procedures. The time to diagnosis varied from almost immediately after the procedure, most likely trauma-related AVF, to more than four years later, when AVF was probably due to chronic venous occlusion and angiogenesis. In our case, approximately two years had passed from the time of catheterization and cardiac device implantation to symptom development, and a further two years before AVF was diagnosed.

Venous thrombotic stenosis related to cardiac device implantation is estimated to occur at a rate of $11 \%(5-25 \%)$ [4], higher if there is infection [8], but it is usually chronic and asymptomatic, and is typically managed conservatively in the absence of symptoms. Around 1-3\% of patients with severe occlusion become symptomatic and require treatment, which generally consists of anticoagulation and endovascular recanalization [4]. In two review articles of a total of 130 patients with cardiac device-related upper limb venous occlusion there were no reported cases of AV fistula [4, 8]. The precise pathophysiology of AV fistula formation due to chronic venous thrombosis remains unclear, but it most 

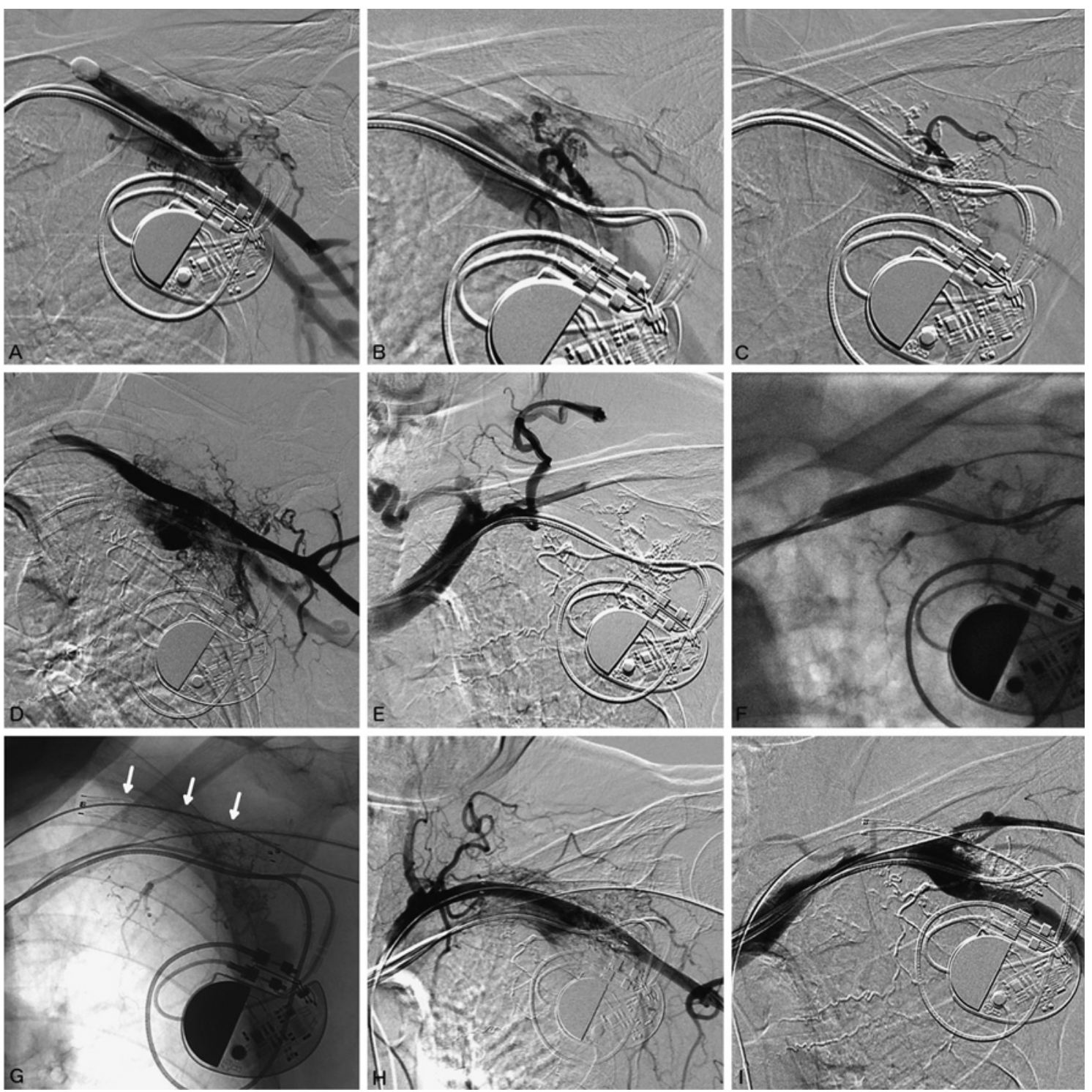

Figure 2. Angiography and interventional procedure for subclavian arteriovenous fistula. A Multiple small shunts into the subclavian vein, venous occlusion between the clavicle and first rib, and retrograde venous flow. B Isolated feeder branch between the subclavian artery and vein. C Postembolization of the feeder branch using $50 \%$ N-butyl cyanoacrylate (NBCA). D Multiple shunts with reduced flow and retrograde venous flow to the axillary vein remaining after embolization. $\mathbf{E}$ Complete occlusion of the left subclavian vein (femoral approach). F Venous angioplasty with non-compliant balloons (Mustang $4 \times 20 \mathrm{~mm}$ at $10-22 \mathrm{~atm}$, and $6 \times 20 \mathrm{~mm}$ at 12-22 atm); the lesion was crossed via the cephalic vein, and then a through-and-through guidewire was established. G Stent graft (arrows) placed in left subclavian artery (via left femoral approach with 9-F sheath). H Subclavian arterial DSA (early phase) showing minimized flow through the remaining arteriovenous shunts. I Subclavian venous DSA (cephalic approach) showing restoration of antegrade venous flow.

probably involves venous hypertension and hypoxiastimulated local angiogenesis, with new small vessels eventually reaching the adjacent artery [9].

There are a number of treatment options for AVFs, including surgical ligation, embolization with coils, ethanol or other agents, sclerotherapy, and stent graft insertion, and a conservative approach may be considered if the patient is asymptomatic [5, 6]. Embolization with ethanol causes endothelial damage and thrombus formation, leading to vascular occlusion, and is generally quite effective $(68-96 \%)$ re- garding symptoms and lesion size $[3,6]$. However, irrespective of the agent used, embolization alone may not be sufficient for a complex defect that involves many small vessels, such as in our case. Stent graft insertion has recently become the favored approach for treatment of traumatic AVFs $[7,10]$, particularly in areas that would involve complicated anatomical exposure and high risk if managed with surgical ligation.

In a retrospective review of 10 cases of AVF formation due to lower limb deep venous thrombosis (DVT) [2], of the 

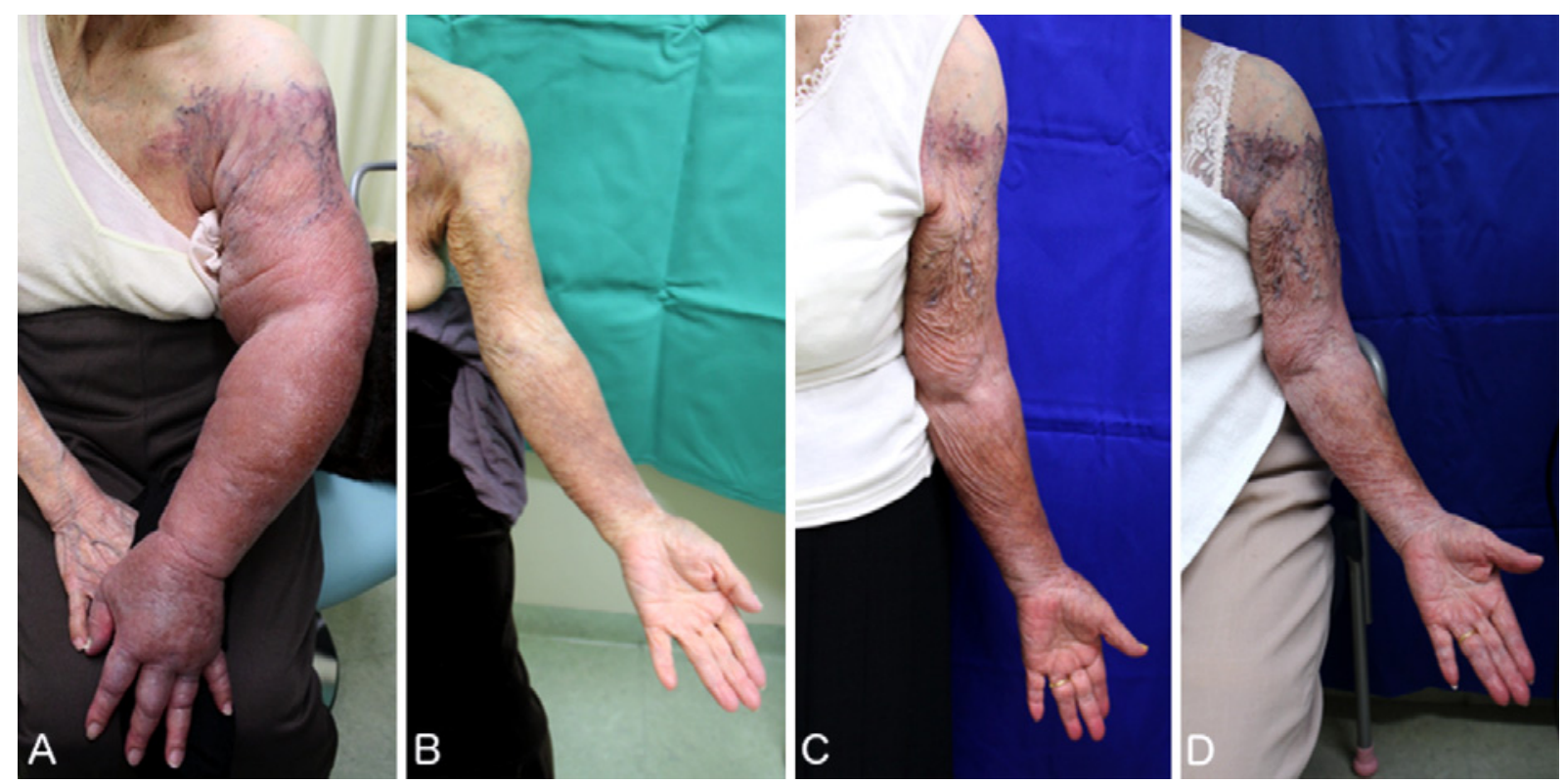

Figure 3. Sequential clinical appearance of the left upper limb affected by subclavian arteriovenous fistula. A Pre-endovascular management with embolization, balloon angioplasty, and stent graft insertion. B Two weeks post-procedure. C Nine months post-procedure: gradual recurrence of venous insufficiency. D 2.5 years following the first angiography (most recent image), including three further venoplasty procedures during this time.

seven patients who showed clinical improvement, four were treated medically, one with stent graft insertion, one with coil embolization, and one surgically with fistula ligation. The technical characteristics of the procedures and the sequelae of the three patients who did not improve were not reported. Link et al. described the management of two cases of acquired lower limb AVF due to chronic DVT [3]. In both cases the AVF was closed by catheterization and embolization of feeder arterial branches with agents including ethanol, alcohol, oil, and coils. In our case there were many feeder branches, so that even after embolization of five branches with NBCA there was still flow through remaining feeder branches. Therefore, the decision was made to use a stent graft to prevent inflow.

In previously reported lower limb cases the underlying thrombotic occlusions were addressed with anticoagulation. Since the patient in our case was warfarinized at the time of fistula formation, the presence of the permanent cardiac pacemaker as the likely underlying etiology of the thrombotic occlusion adds complexity and probably increases the risk of recurrence.

\section{Conclusion}

This case presents acquired AVF formation as a rare but possible long-term complication of chronic subclavian vein thrombotic stenosis after pacemaker insertion. The patient in this case was treated endovascularly with embolization, balloon angioplasty, and stent graft insertion for closure of the fistula and recanalization of the underlying venous obstruction.

\section{Statement of Informed Consent}

The patient involved in this case has provided informed consent to its publication and to the use of the images.

Conflict of interest: The authors have no conflicts of interest to declare in relation to this manuscript.

\section{References}

1. Sato O, Tada Y, Sudo K, Ueno A, Nobori M, Idezuki Y. Arteriovenous fistula following central venous catheterization. Arch Surg 1986; 121: 729-731.

2. Elias A, Bourrinet A, Elias M, Rousseau H. Lower limb angiogenesis with arterio-venous fistulas, a new entity aggravating the long-term clinical course of deep vein thrombosis. Thromb Haemost 2004; 92: 214-217.

3. Link DP, Garza AS, Monsky W. Acquired peripheral arteriovenous malformations in patients with venous thrombosis: report of two cases. J Vasc Interv Radiol 2010; 21: 387-391.

4. Rozmus G, Daubert JP, Huang DT, Rosero S, Hall B, Francis C. Venous thrombosis and stenosis after implantation of pacemakers and defibrillators. J Interv Card Electrophysiol 2005; 13: 9-19.

5. Hyodoh H, Hori M, Akiba H, Tamakawa M, Hyodoh K, Hareyama M. Peripheral vascular malformations: imaging, treatment approaches, and therapeutic issues. Radiographics 2005; 25 Suppl 1: S159-171.

6. Do YS, Yakes WF, Shin SW, et al. Ethanol embolization of arteriovenous malformations: interim results. Radiology 2005; 235 : 674-682.

7. Parodi JC, Schonholz C, Ferreira LM, Bergan J. Endovascular stent-graft treatment of traumatic arterial lesions. Ann Vasc Surg 
Interventional Radiology 2017; 2: 33-37

1999; 13: 121-129.

8. Li X, Ze F, Wang L, et al. Prevalence of venous occlusion in patients referred for lead extraction: implications for tool selection. Europace 2014; 16: 1795-1799.

9. Kubis N, Levy BI. Understanding angiogenesis: a clue for under- standing vascular malformations. J Neuroradiol 2004; 31: 365-368.

10. Hagspiel KD, Komorowski DJ, Shih MC, Peeler BB, Jensen ME.

Treatment of carotid arteriovenous fistula with balloon-expandable tracheobronchial covered stent. J Vasc Interv Radiol 2006; 17: 585-586. 